\title{
Large-Eddy-Simulation Prediction of an Installed Jet Flow and Noise with Experimental Validation
}

\author{
Zhong-Nan Wang* \\ University of Cambridge, Cambridge, England CB2 1PZ, United Kingdom \\ Anderson Proenca $₫$ and Jack Lawrence \\ University of Southampton, Southampton, England SO17 1BJ, United Kingdom \\ Paul G. Tucker \\ University of Cambridge, Cambridge, England CB2 1PZ, United Kingdom \\ and \\ Rod Self \\ University of Southampton, Southampton, England SO17 1BJ, United Kingdom \\ https://doi.org/10.2514/1.J058921
}

\begin{abstract}
Extra noise is generated when jets are installed beneath solid surface. In this paper, large-eddy simulation (LES) has been used to investigate these jet installation effects. The simulations were performed on two jet configurations: an isolated jet and a jet installed below a horizontal flat plate. The LES is compared with experimental data from measurements using hot wires, unsteady surface pressure sensors, and far-field microphones. Good agreement has been achieved between LES and experimental measurements on flow statistics, space-time correlations, and both near-field and far-field sound spectra. The installed effects are discussed from the perspective of flows and acoustics in the validation process.
\end{abstract}

3.

$A_{i j}^{c, n}$
$c_{0}$
$D$
$F_{i j}^{c, n}$
$H$

$L$
$M_{j}$
$Q_{i}$
$R_{i j}$
$r$
$S t$
$U, V$
$U_{j}$
$u_{i}$
$W$
$\boldsymbol{x}$
$\zeta$
$\tau$
$\tau_{i j}$
$\phi$

Jacobi matrix of $F_{i j}^{c, n}$

sound speed of ambient fluids, $\mathrm{m} / \mathrm{s}$

nozzle diameter, $\mathrm{m}$

numerical convective flux between control volume $i$ and $j$

distance between nozzle center line and plate lower surface, $\mathrm{m}$

distance between nozzle exit and plate trailing edge, $\mathrm{m}$ jet Mach number, $U_{j} / c_{0}$

conservative variables at control volume $i,[\rho, \boldsymbol{u}, \rho E]$

space-time correlation of velocities $u_{i}$ and $u_{j}$

radial distance from nozzle center line, $\mathrm{m}$

Strouhal number

time-averaged axial, radial velocity, $\mathrm{m} / \mathrm{s}$

maximum axial velocity along nozzle center line, $\mathrm{m} / \mathrm{s}$

instantaneous velocity in the direction $i, \mathrm{~m} / \mathrm{s}$

half plate width, $\mathrm{m}$

coordinates $[x, y, z], \mathrm{m}$

spatial separation distance $[\xi, \eta, \phi], \mathrm{m}$

time delay, $\mathrm{s}$

Reynolds stress tensor

observer polar angle, deg

Subscript

$j \quad=$ jet

Presented as Paper 2019-2435 at the AIAA Aeroacoustics Conference, Delft, The Netherlands, May 20-30, 2019; received 2 August 2019; revision received 20 December 2019; accepted for publication 9 February 2020; published online XX epubMonth XXXX. Copyright (ङ 2020 by The Authors. Published by the American Institute of Aeronautics and Astronautics, Inc., with permission. All requests for copying and permission to reprint should be submitted to CCC at www.copyright.com; employ the eISSN 1533-385X to initiate your request. See also AIAA Rights and Permissions www.aiaa.org/randp.

*Research Associate, Department of Engineering. Member AIAA.

${ }^{\dagger}$ Research Fellow, Institute of Sound and Vibration Research. Member AIAA.

*Rank Professor of Engineering, Department of Engineering. Associate Fellow AIAA.

${ }^{\S}$ Professor of Aeroacoustics, Institute of Sound and Vibration Research. Member AIAA.

\author{
Superscripts \\ $c \quad=$ convective \\ $n \quad=$ surface normal
}

\section{Introduction}

S GLOBAL air traffic volume continues to grow, currently by $A$ about $8 \%$ per year, aircraft noise becomes of great concern to residents living around airports. Jet noise from aeroengines is still one of the dominant components when an aircraft is taking off. High bypass ratios, used to increase engine efficiency, significantly reduces jet noise by decreasing the effective jet velocity $U_{j}$, as jet mixing noise scales with $U_{j}^{8}[1]$ ]. However, the increased bypass ratio enlarges engine diameters, which in turn results in the engine installation much closer to the wing. This intensified jet-wing interaction introduces a significant low-frequency noise source, referred to as installed jet noise [2]. Especially for the next-generation ultra-highbypass-ratio (UHB $\bar{R}$ ) aeroengine, the small gap between the engine and wing will further increase installed jet noise. It is, therefore, important to predict, understand, and then reduce installed jet noise.

In over 60 years of research, considerable progress has been made on isolated jet noise. However, the understanding of installed jets and their acoustics is still limited. Historically, the extra noise due to the wing installation was first noticed by Bushell [3] when comparing an in-flight installed jet with a static isolated jet for a full-scale aircraft. The acoustic characteristics of installed jets have been extensively studied in the mid-1970s by experimentalists $[2,4]$. The directivity of installation noise is found to be dipole-like and be sensitive to the distance from the jet center line to the solid surface and from the jet exit to the surface trailing edge (TE). To understand the noise mechanism, Ffowcs Williams and Hall observed that the far-field low-frequency noise is amplified when a turbulent flow is installed close to a surface [5]. They attributed this amplification to the scattering of the hydrodynamics pressure field near the solid surface edge. Lyu et al. confirmed this theory and developed a reliable prediction model [6] based on the hydrodynamic wave scattering. This model demonstrated that the far-field sound amplification can be accurately predicted by incorporating the scattering of near-field hydrodynamic evanescent waves using Amiet's approach [7]. However, comprehensive unsteady flow data of installed jets are currently 
not available for development and validation of low-order acoustic source models. High-fidelity numerical simulation is therefore used to fulfill this mission, because it is relatively difficult to achieve timeaccurate flow measurements.

Large-eddy simulation (LES), as a high-fidelity simulation method, has been successfully used to predict the noise generated from isolated turbulent jet flows [8]. Recent growth of computational power and development of numerical algorithms has enabled the simulation of industrial-level propulsive jets with complex geometries by resolving large-scale turbulent motions [9]. Recently, the flow and noise from an UHBR engine installed under a wing was well simulated using a hybrid LES-Reynolds-averaged Navier-Stokes (RANS) method [11]. The far-field noise was successfully predicted and agreed with the far-field acoustic measurements. However, it is difficult to completely separate the overall installation effects for such complex configuration, including the scattering of the linear hydrodynamic instability wave, the changes of jet mixing noise source, and any effects due to unsteady wing lift. In this context, simulations on simplified canonical cases are necessary in order to isolate these installation effects and to better understand the individual noise mechanisms. Furthermore, near-field flow and pressure measurements also exist to validate such numerical computations in detail.

In this paper, LES has been performed on two jet configurations: an isolated jet and a jet installed below a flat plate. The plate is placed in the linear hydrodynamic region of the jet flow [10], which avoids nonlinear flow interactions. This is intended to include the installation noise only from the scattering of hydrodynamic instability waves. The simulation data have been compared with the experimental measurements following the validation metrics proposed by Bridge and Wernet [12]. The installation effects are then discussed from the aerodynamic and acoustic points of view.

\section{Jet Configurations and Operating Conditions}

To isolate jet installation effects, a canonical case was designed to be a single stream jet beneath a flat plate. The configuration is shown in Fig. 1 with a three-dimensional view of an $x-y$ plane for jet flows and acoustics with coordinates system illustrated. Three parameters are used to control the position of the jet relative to the flat plate: $H$ represents the vertical distance from jet nozzle center line to plate surface, and $L$ represents the distance from nozzle exit plane to plate TE. The nozzle is located in the middle span of the plate, and $W$ is the half span length. The jet operates at a Mach number of 0.6 , which is the typical bypass flow speed of future UHBR aeroengines. The Reynolds number based on nozzle diameter and jet velocity is around $7 \times 10^{5}$. For this configuration, the jet flow barely scratches the TE of the plate without touching the plate underneath surface. Flow impingement is avoided, such that the downstream jet mixing noise sources remain unaffected. Additional noise source is introduced by the scattering of linear hydrodynamic wave from the plate TE. For comparison, an isolated jet was chosen with the same nozzle geometry and is operated at the same flow Mach number. The operating conditions and jet configurations are summarized in Table 1 .

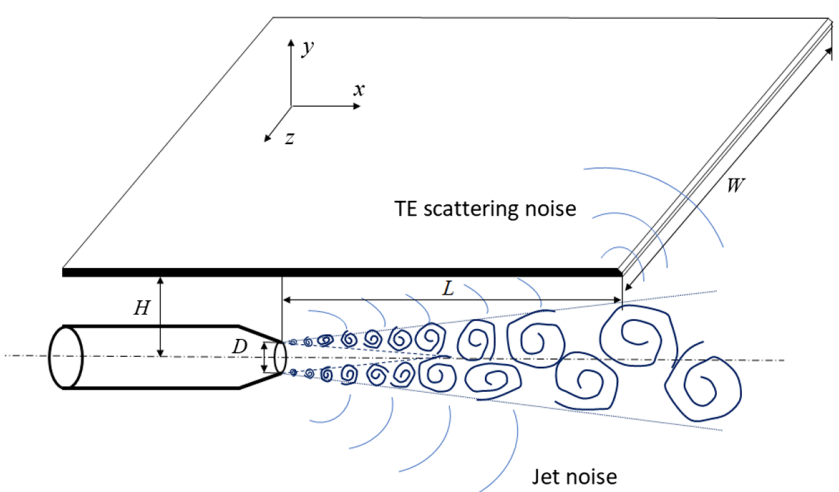

Fig. 1 Installed jet configuration with an $x$ - $y$ plane cutting through the plate and jet flow.
Table 1 Jet configuration and operating conditions

\begin{tabular}{lcccc}
\hline \hline Jet configuration & $M_{j}$ & $L / D$ & $H / D$ & $W / D$ \\
\hline Installed jet & 0.6 & 4 & 1 & 7 \\
Isolated jet & 0.6 & - & - & - \\
\hline \hline
\end{tabular}

\section{Numerical Methods}

\section{A. Flow Solver}

The solver [9] is a compressible, finite volume, and edge-based solver using dual meshes. It solves the unsteady Navier-Stokes equations on unstructured meshes and, hence, is capable of modeling complex geometries of any shape using a variety of cell topologies. The spatial discretization is achieved using a second-order kineticenergy-preserving (KEP) scheme [13] with a fourth-order numerical smoother adapted for convective fluxes:

$$
F_{i j}^{c, n}=F_{i j, \mathrm{KEP}}^{n}-\frac{1}{2} \varepsilon\left|A_{i j}^{c, n}\right|\left[L\left(Q_{j}\right)-L\left(Q_{i}\right)\right]
$$

where $F_{i j, \mathrm{KEP}}^{n}$ is the KEP fluxes $\left[\bar{\rho} \bar{u}^{n}, \bar{\rho} \overline{\boldsymbol{u}} \bar{u}^{n}+\bar{p} \boldsymbol{n}, \bar{\rho} \bar{H} \bar{u}^{n}\right]$, and the overbar represents the arithmetic averaging operator using the values in the adjacent mesh elements $i$ and $j$. The KEP scheme itself is numerically stable and nondissipative, which is suitable for LES computation. Previous tests using homogeneous decaying turbulence with hexahedral, tetrahedral, and prism elements showed that the KEP scheme was less sensitive to grid types, which enables LES to be performed on hybrid structured-unstructured meshes. The second term in Eq. (1) is the numerical smoother. The parameter $\varepsilon$ is used to control numerical smoothing in the simulation. This is minimized in the jet flow zone and is increased along with the grid expansion to form a sponge zone near the boundaries to prevent wave reflections. Second-order backward differencing is used for physical time marching. Implicit physical time stepping is made possible through the use of dual time stepping. A fourth-order Runge-Kutta method is used in each pseudo-time step with $C F L=1$. The physical time step is $0.0016 \mathrm{D} / U_{j}$. The total sampling time is $293 \mathrm{D} / U_{j}$.

LES is used to resolve the turbulent jet plume with underresolved scales modeled by the WALE SGS model [14]. Boundary layers near the nozzle wall are modeled using the standard Spalart-Allmaras (SA) RANS turbulence model [15]. The RANS stress tensor $\tau_{i j}^{\text {RANS }}$ is hybridized with the SGS stress tensor $\tau_{i j}^{\mathrm{SGS}}$ using a blending function $f$ based on modified wall distance [9].

$$
\tau_{i j}^{\mathrm{turb}}=f \tau_{i j}^{\mathrm{SGS}}+(1-f) \tau_{i j}^{\mathrm{RANS}}
$$

Turbulent flow profiles are therefore generated inside the nozzle by RANS without turbulent velocity fluctuations in streak structures resolved near the wall. The steep velocity gradient of turbulent profiles introduces strong Kelvin-Helmholtz instabilities, which naturally trigger rapid transition into three-dimensional structures downstream of the nozzle lip within $0.1 D$. No significant effects on near/far-field acoustics are observed except a tonal component introduced by the transition process. The solver has been extensively validated on a range of jet flows [9]. Parallel performance is achieved using MPI and ParMetis grid partitioning, showing almost linear scaling over 10,000 cores. The parallel I/O is also made efficient by using HDF5 library, and each core can visit the same file simultaneously without blocking.

\section{B. Computational Domain and Mesh}

Figure 2 shows the computational domain. It is of conical shape with a radial size of $30 D_{j}$ at the inlet plane at $x=-15 D_{j}$, and $50 D_{j}$ at the outlet plane at $x=100 D_{j}$. It contains two zones: LES and sponge zones. The LES zone starts from $3 D_{j}$ upstream and extends to $30 D_{j}$ downstream of the nozzle exit in the axial direction, with a radial size of $7 D_{j}$. The rest of the domain is filled with sponge zones, where a high level of smoothing is applied to reduce any numerical reflections from computational boundaries. Total temperature and pressure are 


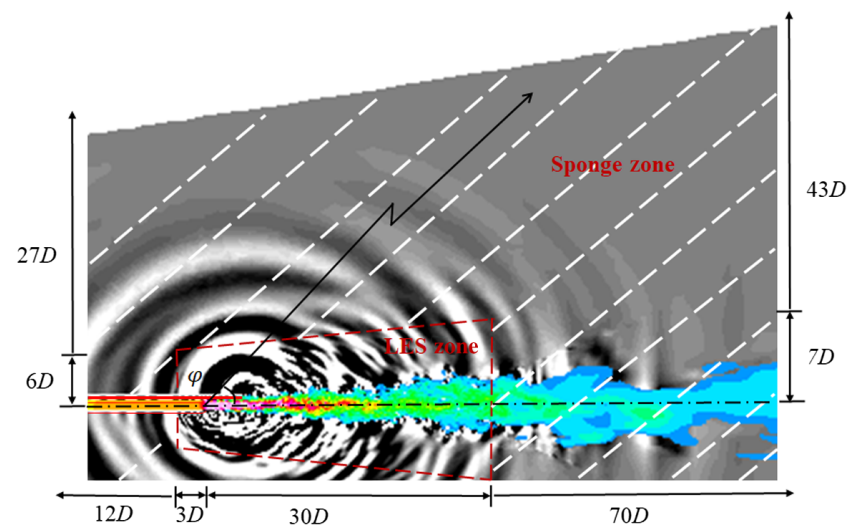

Fig. 2 Computational domain of jet simulation.

Table 2 Grid spacing distribution along lip line

\begin{tabular}{lrrrrrrr}
\hline \hline$x / D$ & \multicolumn{1}{c}{0} & \multicolumn{1}{c}{1} & \multicolumn{1}{c}{2} & \multicolumn{1}{c}{5} & \multicolumn{1}{c}{10} & 20 & 30 \\
\hline$D / d x$ & 80 & 75 & 70 & 50 & 35 & 20 & 10 \\
$D / d r$ & 591 & 450 & 350 & 200 & 100 & 60 & 50 \\
\hline \hline
\end{tabular}

imposed at the nozzle inlet. Far-field conditions are specified at the upstream boundary and side cylindrical boundary surfaces, whereas static pressure is specified at the downstream boundary.

Hexahedral cells are used for the isolated jet simulation. The grid line aligns with the shear-layer trajectories to provide better resolution. The grid gradually expands in the axial direction following jet spreading rate. The mesh resolution along the lip line is shown in Table 2. The $y^{+}$is around 1 near the nozzle wall, and $x^{+}$is around 7 at the nozzle exit. For the installed jet simulation, the same hexahedral cells are maintained in the jet plume. The plate surface is meshed with a quad-dominant mesh from where prism layers are extruded. Further refinement on the plate surface mesh is made near the nozzle and jet plume because the small-scale turbulence and high-frequency acoustics are generated there. Outside of the jet plume, tetrahedral cells are used to resolve the near-field acoustics. The position of the interface between the hex-meshed jet plume zone and the tet-meshed acoustics zone is carefully adjusted. The adjustment is based on the criterion that the aspect ratio of the hexahedral mesh on the interface is close to 1 , as the tetrahedral cells connected to the interface are isotropic. The interface radius $R$ is chosen to be $R \Delta \theta \approx \Delta R \approx \Delta x$. This makes a smooth transition in size from jet plume hexahedral mesh to acoustics tetrahedral mesh. The hybrid mesh is shown in Fig. 3. The mesh
Table 3 Mesh statistics $\left(\times 10^{6}\right)$

\begin{tabular}{lcllcr}
\hline \hline & $N_{\text {tex }}$ & $N_{\text {pyr }}$ & $N_{\text {prism }}$ & $N_{\text {hex }}$ & $N_{\text {cell }}$ \\
\hline Isolated jet & 0 & 0 & 0 & 34.94 & 34.94 \\
Installed jet & 30.46 & 0.26 & 0.02 & 17.89 & 48.64 \\
\hline \hline
\end{tabular}

statistics is listed in Table 3 . The current grid density is chosen according to our best practice guidelines [16] obtained by simulating a large number of jets from canonical to industrial complexity levels. The grid independence and azimuthal grid spacing sensitivity have been tested in previous research [17]. The total number of grid points varied from 18 to 50 million, and the azimuthal grid number varied from 120 to 180. They showed the gird-independent results in flow statistics and far-field acoustics. This grid independence is also confirmed by other researchers [18], from 10 to 64 million. Therefore, the current mesh size should be enough to fulfill the task in the current simulation.

\section{Far-Field Sound Prediction}

Sound at far-field observer locations is predicted by performing surface integration at a retarded time, which accounts for the propagation time spent from source to observer, following the Ffowcs Williams-Hawkings (FW-H) equation. A general convective FW-H formulation [19] is used in this paper by setting ambient fluid velocity to zero. The volume integration is removed by enclosing all the quadrupole sources within the FW-H surfaces. The FW-H surfaces are placed in the irrotational hydrodynamic region to prevent any nonlinear hydrodynamic contaminations on acoustic predictions. The placement follows the proposed criterion: turbulent intensity less than $0.25 \%$ and normalized vorticity magnitude less than $0.05 \%$ at the FW-H surfaces [9].

Figure 4a shows the FW-H surface placement in the flow and acoustic field of installed jets. To assure a robust and convergent farfield sound prediction, three sets of FW-H surfaces (shown in blue, red, and green) are placed at different radial distances in the irrotational region away from turbulent jet plume. Axisymmetric conical surfaces are used for the isolated jet, whereas additional surfaces are devised around the flat plate for installed jets to enclose the extra sound sources introduced by jet-surface interactions. Figure $4 \mathrm{~b}$ shows that the conical surfaces merge with the plate-wrapping surfaces, forming the complete set of FW-H surfaces for the installed jet. Unstructured elements are used to discretize the FW-H surfaces. In the simulation, unsteady flow data are recorded on the centroids of these surface elements for acoustic postprocessing. This far-field sound prediction procedure has been widely tested in our previous jet noise simulations $[\underline{9}, \underline{11}]$.
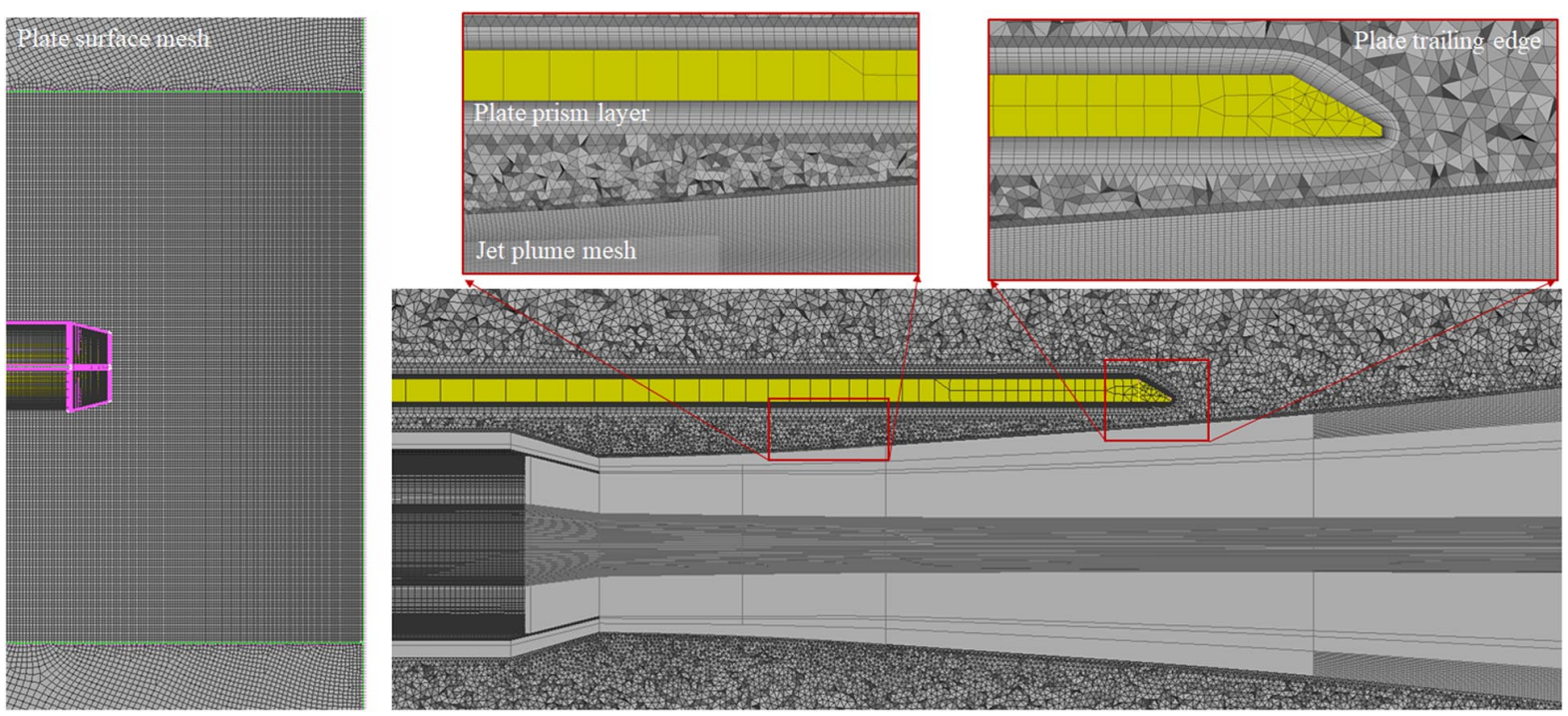

Fig. 3 Computational mesh of the installed jet. 


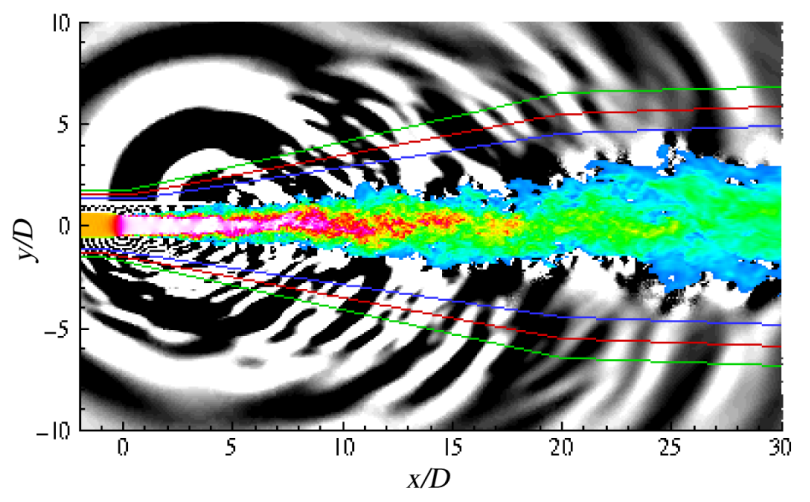

a) FW-H surfaces in flow and acoustic fields

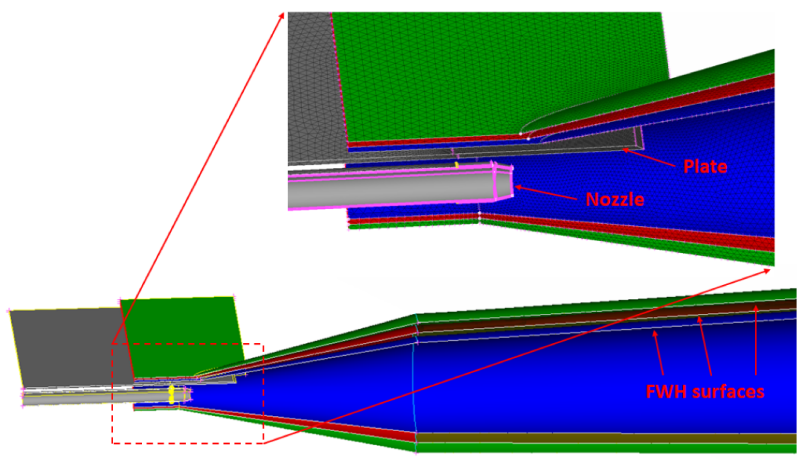

b) FW-H surface configurations

Fig. 4 Placement of three sets of FW-H surfaces (in blue, red, and green) for the installed jet.

\section{Experimental Techniques}

The experiments were performed in the anechoic Doak Laboratory (DOAK) within the Institute of Sound and Vibration Research, at the University of Southampton. The DOAK jet facility is approximately $11 \mathrm{~m} \times 7 \mathrm{~m} \times 6 \mathrm{~m}$ in size and is anechoic down to $400 \mathrm{~Hz}$, shown in Fig. 5 .

Near-field acoustic measurements were made using a traversable azimuthal near-field array, containing eight equally spaced $1 / 4$-inch microphones. They were positioned at eight different axial locations along the jet $(0 \leq x / D \leq 7)$. Both polar and azimuthal measurements of the acoustic far-field were performed using a semicircular traversable array populated with five equally spaced microphones at $45^{\circ}$ intervals located at a minimum distance of $50 \mathrm{D}$ from the nozzle exit (i.e., when the array was positioned at $\phi=90^{\circ}$ ). The polar observer angles ranged between $40^{\circ} \leq \phi \leq 130^{\circ}$ at $10^{\circ}$ intervals. The far-field acoustic data were corrected to 1 -m-lossless values to account for spherical propagation and atmospheric attenuation [20]. More details about the acoustic test setup can be found in the thesis of Lawrence [21].

Velocity measurements were carried out using a constant-temperature anemometry (CTA) system. Dantec 55P11 single probes were used to record the unsteady velocity. Axial and radial traverses using only one sensor were performed to survey the jet single-point statistical moments. To measure the joint moments, two hot-wire probes were mounted on two independent traverse systems. A more detailed description of the CTA system, the traverse setup, and the instrumentation used in the aerodynamic experiments are provided by Proenca [22] and Proenca et al. [23].

\section{Results}

\section{A. Overview of Flowfield}

Figure 6 shows instantaneous Q-criterion surfaces of the installed jet with acoustic waves in the background from the LES. The flow

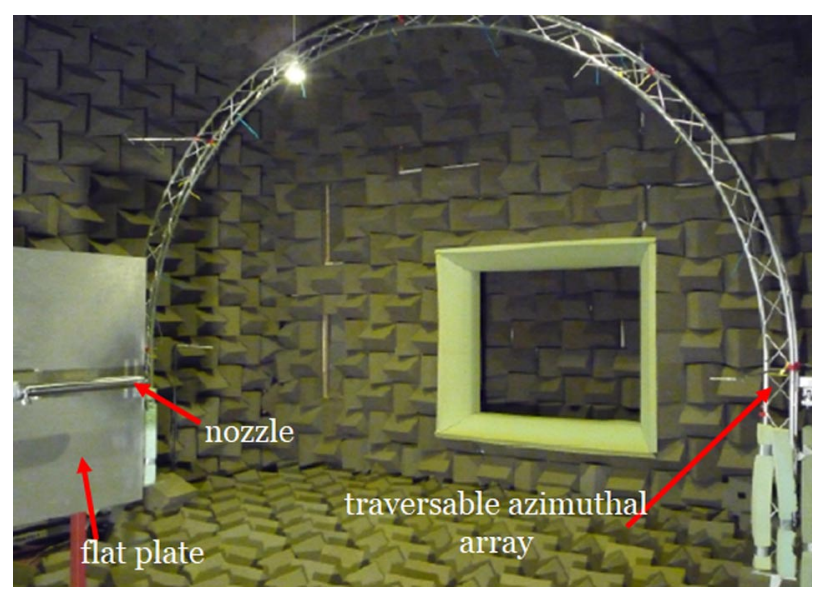

Fig. 5 Anechoic Doak Laboratory jet facility. and acoustics are well resolved in the simulation. Hairpin vortices roll up in the jet plume and grown in size as they move downstream. When a plate is installed near the jet, interactions occur in both hydrodynamics and acoustics. The hydrodynamic interactions are relatively weak for the current configuration: the installed jet flow slightly scrapes the plate TE without significant modification of flow structures, as seen in the close-up view. This weak impact on jet hydrodynamics will be further analyzed in the sections of flow statistics and space-time correlations by comparison with the isolated jet. However, strong interactions are observed in acoustics. First, jet noise generated by shear layer near the nozzle is shielded above and reflected under the plate. Second, a new sound source is introduced around the TE. This is because near-field evanescent hydrodynamic waves are scattered by the plate TE and converted into propagating acoustics. The TE scattering noise propagates into the forward arc, as seen in Figs. $\underline{4 a}$ and $\underline{6}$. These acoustic effects will be discussed quantitatively in the sections of near-field spectra and far-field acoustics.

\section{B. Flow Statistics}

Figure $\underline{7}$ shows the radial profiles of both isolated and installed jet mean velocities at different axial locations in the upper $x-y$ plane, where the difference between installed and isolated jets is available. The LES prediction achieves a good agreement with the axial component of velocity measured by the hot wires, shown in Fig. 7a. The plate installation appears to have a negligible effect on axial velocity. A slight acceleration is seen to occur around the plate

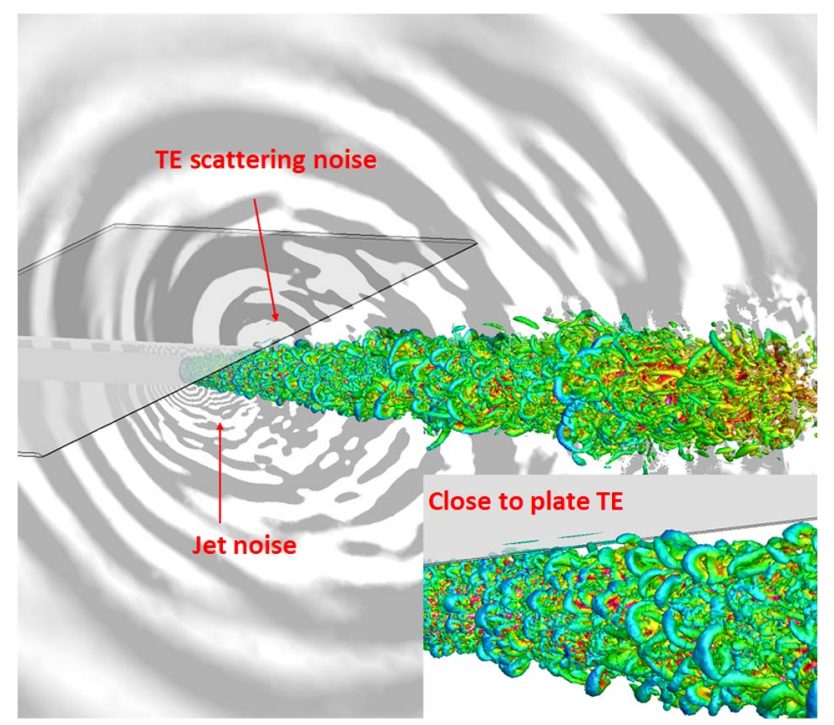

Fig. 6 Instantaneous flow and acoustics in installed jets from the LES: Q-criterion isosurfaces colored by axial velocity with time derivative of pressure as background contours. 

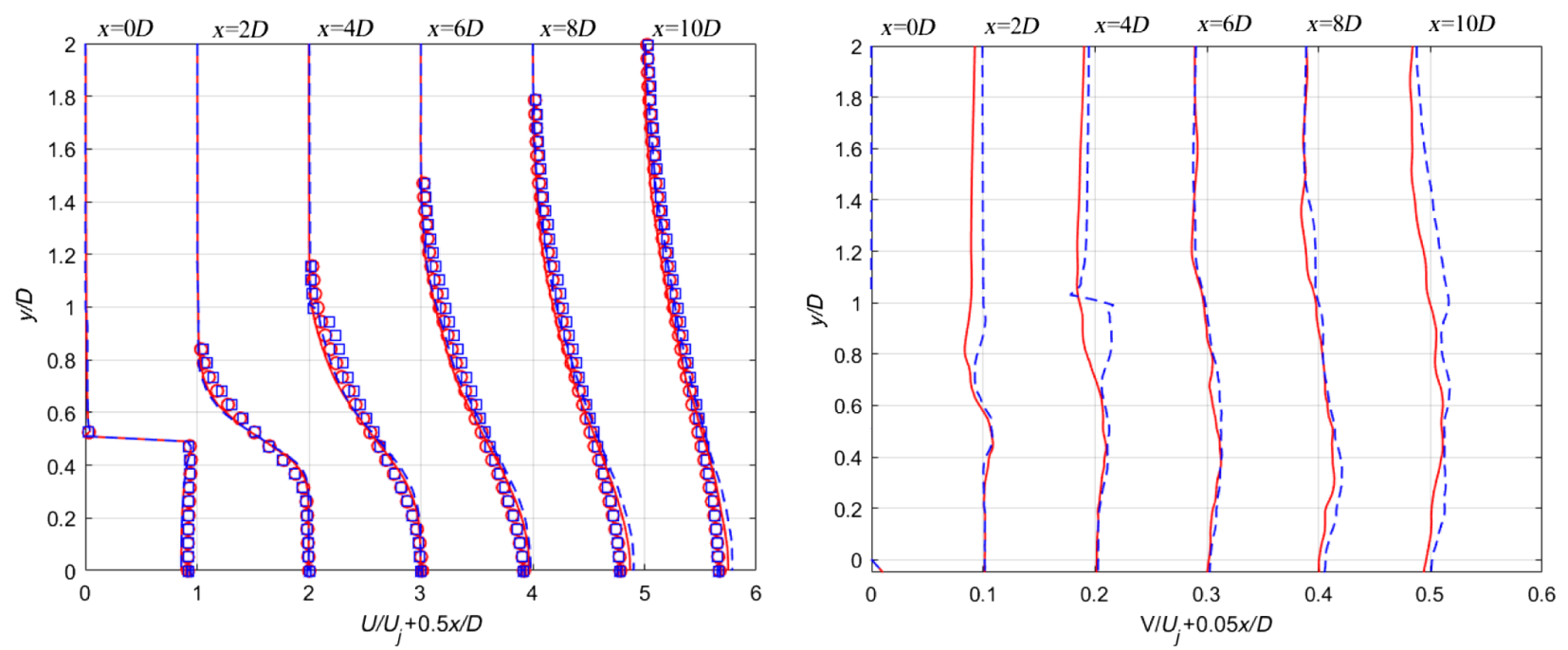

a) Axial velocity

b) Radial velocity

Fig. 7 Radial profiles of time-averaged velocities at $x / D=\mathbf{0 , 2 , 4 , 6 , 8}$, and 10 for both isolated and installed jets: red circles, hot-wire data for the isolated jet; blue squares, hot-wire data for the installed jet; red solid lines: LES data for the isolated jet; blue dashed lines: LES data for the installed jet.

TE (at $\left.x / D_{j}=4\right)$ and immediately downstream of the TE in Fig. 7 a. Figure $7 \mathrm{~b}$ shows the radial velocity profiles at various axial locations. Negative radial velocity out of jet plume $(r / D>1)$ indicates that ambient flows are entrained into the jet plume for the isolated jet. For the installed jet, the radial velocity above the plate $(x / D<4$ and $r / D>1$ ) becomes zero. The jet entrainment is restricted by the presence of the plate, and a radial expansion occurs around the plate TE $(x / D=4$ and $0.5<r / D<1)$.

Figure $\underline{8}$ shows the radial profiles of the velocity fluctuation rms. Again, the LES agrees well with hot-wire measurements, shown in Fig. 8a. The turbulent fluctuations are slightly suppressed by the plate due to the acceleration seen near the plate TE. The magnitude of the radial fluctuations is also reduced under the plate due to the restricted nature of the flow. Finally, slightly higher turbulence fluctuations are observed downstream of the plate at $x / D_{j}=6,8,10$ compared with the isolated jet. This is caused by the flow acceleration upstream of the TE and the subsequent radial expansion downstream of the plate TE.

\section{Space-Time Correlations}

As suggested by Bridge and Wernet [12], the LES has also been validated by exploring the second-order space-time correlation of velocity field, mathematically defined as

$$
R_{i j}(\boldsymbol{x}, \zeta, \tau)=\frac{\overline{u_{i}^{\prime}(\boldsymbol{x}, t) u_{j}^{\prime}(\boldsymbol{x}+\zeta, t+\tau)}}{\sqrt{\overline{u_{i}^{\prime 2}(\boldsymbol{x})}} \sqrt{\overline{\overline{u_{j}^{\prime 2}(\boldsymbol{x}+\zeta)}}}}
$$

The turbulence integral scales are calculated from these correlations and can be used to inform future turbulence and acoustic modeling. Figure 9a shows the comparison of space-time correlations of axial velocity for different axial separations $\zeta=(\xi, 0,0)$ at $x / D=8$ and $r / D=0.5$, where the experimental measurements are available. For both the isolated and installed jets, the peak locations of each axial separation are well captured by the LES despite the peak value being lower for some of the separations. The underprediction of the peak values could be caused by the short sampling length of the LES (the sampling length is only around 1/100 of the measurement) or the subgrid dissipation. Because these correlations along the nozzle lip line can be collapsed well by scaling with the shear layer width $\delta_{\beta}$ and jet velocity $U_{j}$ [23], experimental measurement at a single axial location is therefore sufficient to validate the simulation quality at different axial locations in the shear layer. Figure $9 \mathrm{~b}$ demonstrates this scaling of correlations $R(x, \zeta=(\xi, 0,0), \tau=\overline{0})$ using shear layer width $\delta_{\beta}$ with the LES data, and a best-fit curve from the isolated jet measurement is shown for reference. Comparing the installed and isolated jets, the plate effects do not appear to affect the

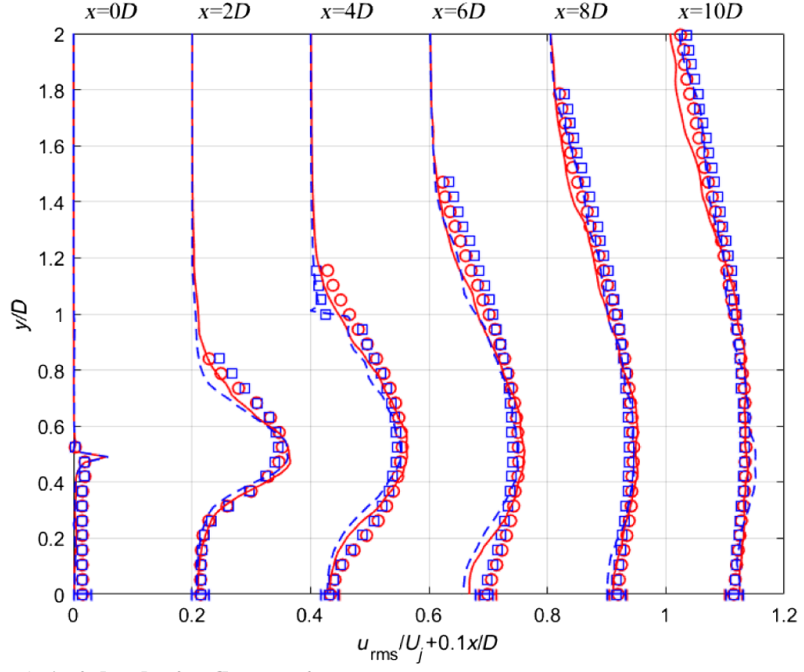

a) Axial velocity fluctuations

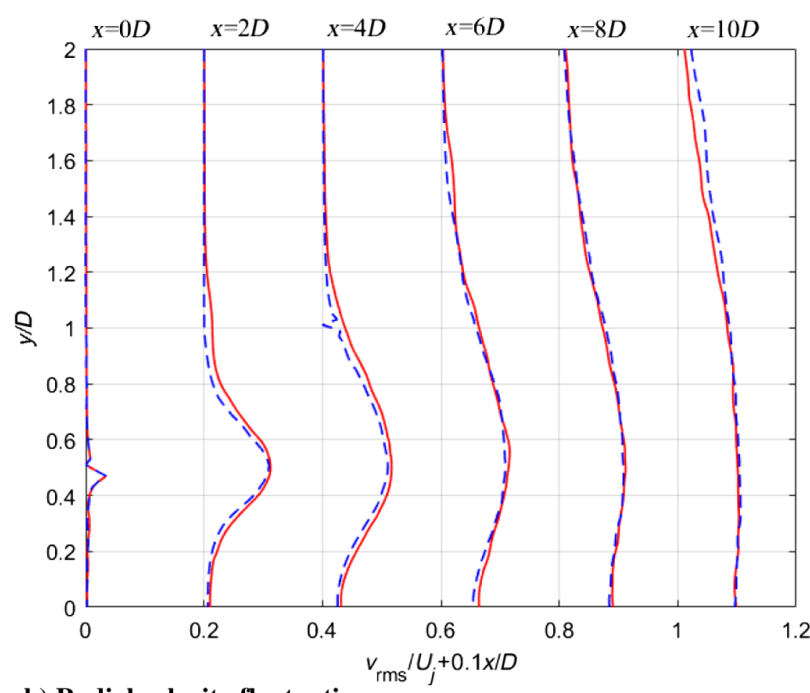

b) Radial velocity fluctuations

Fig. 8 Radial profiles of velocity fluctuations at $x / D=0,2,4,6,8,10$ for both isolated and installed jets: red circles, Hot-wire data for the isolated jet; blue squares, Hot-wire data for the installed jet; red solid lines: LES data for the isolated jet; blue dashed lines: LES data for the installed jet. 


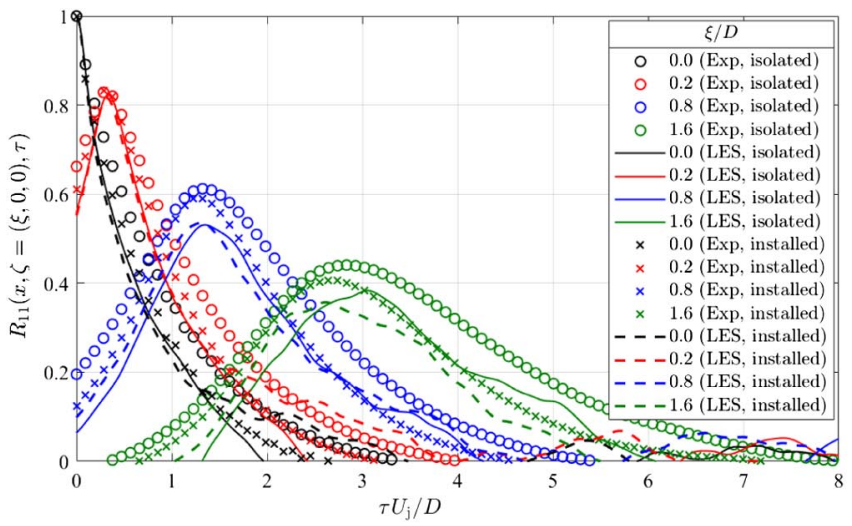

a) $R_{11}$ with axial separations $\xi$ at $x / \mathrm{D}=8$ and $r / \mathrm{D}=0.5$

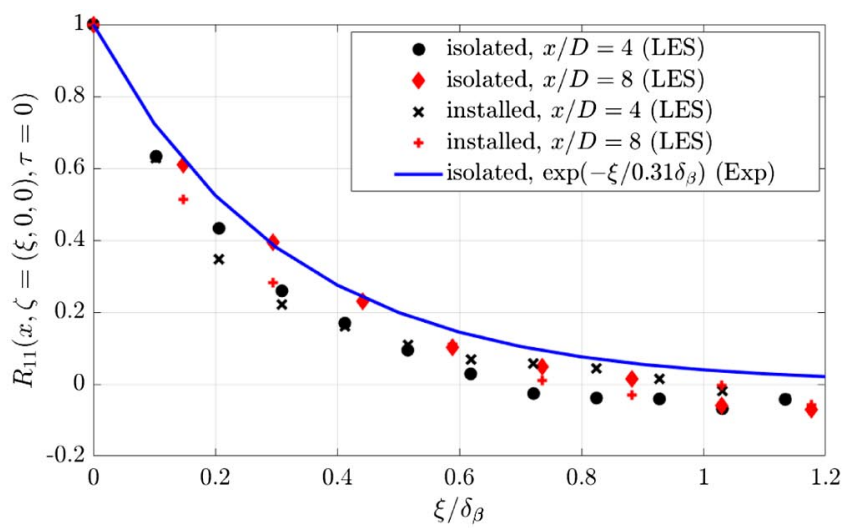

b) $R_{11}$ with axial separations $\xi$ at $\tau=0$

Fig. 9 Comparison of axial-velocity space-time correlation $R_{11}$ between hot-wire and LES data.

jet significantly for this configuration. The correlations at $x / D=4$ and $x / D=8$ collapse onto one curve for both installed and isolated jets and are slightly lower than the experimental best-fit curve. This indicates that the predicted length scale is smaller than the $0.31 \delta_{\text {beta }}$ value provided by the experiment. Overall, an encouraging agreement is found between the hot-wire measurement and the LES prediction of space-time correlations.

To further examine the installation effects on jet turbulence, spacetime correlations are explored for both axial velocities, shown in Fig. 10, and radial velocities, shown in Fig. 11. The reference point is located at $x / D=4$ and $r / D=0.5$ inside the shear layer directly under the plate TE, where the largest differences between the isolated and installed jets are expected. Both axial and radial separations are investigated based on this reference point. For the axial separations, axial and radial velocity correlations of the installed jet increase slightly above that of the isolated before $\xi / D=0.5$. This is because flow accelerates around the plate TE. The velocity correlation decays slightly faster in installed jets for radial separations, because jet spreading is confined by the plate. In general, no significant difference in the turbulent structures is seen between the isolated and

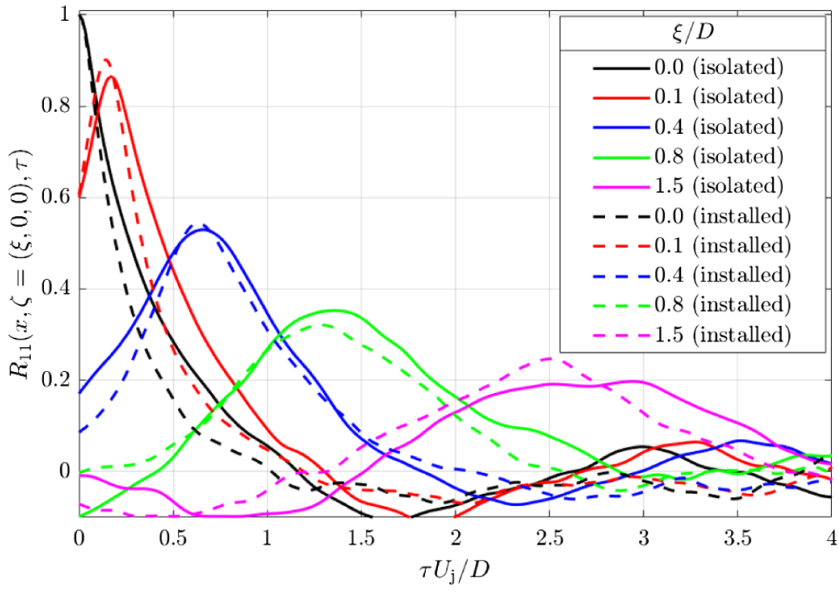

a) Axial separation $\zeta=(\xi, 0,0)$

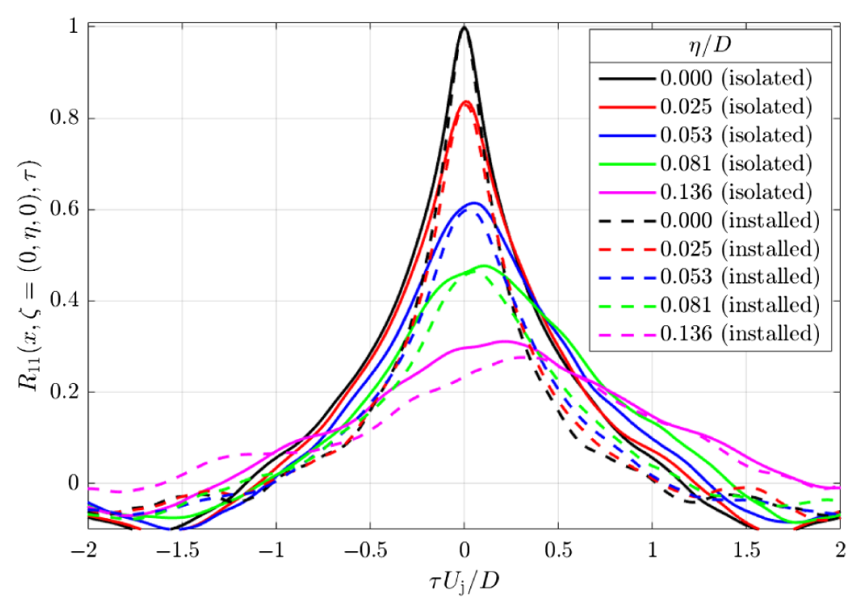

b) Radial separations $\zeta=(\mathbf{0}, \eta, 0$,

Fig. 10 Axial-velocity space-time correlation $R_{11}$ at $x / D=4$ and $r / D=0.5$ for isolated and installed jets.

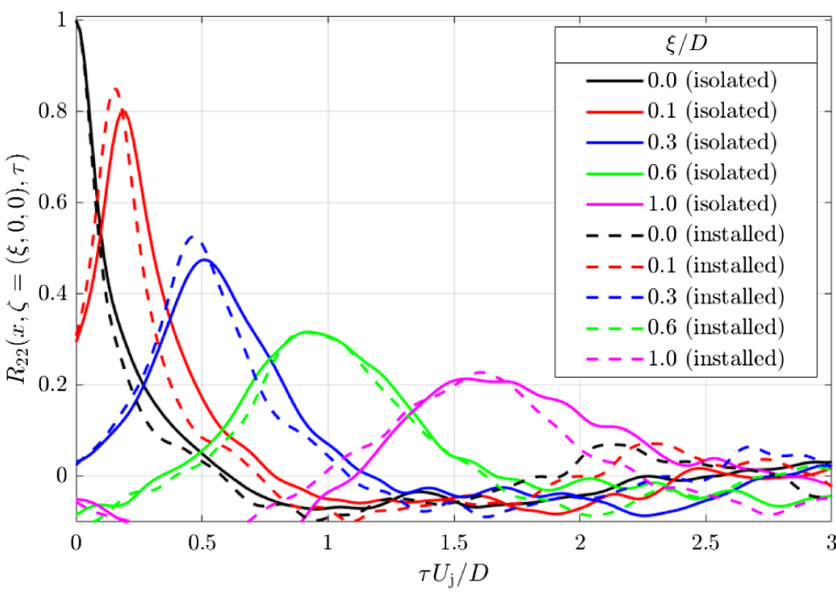

a) Axial separation $\zeta=(\xi, 0,0)$

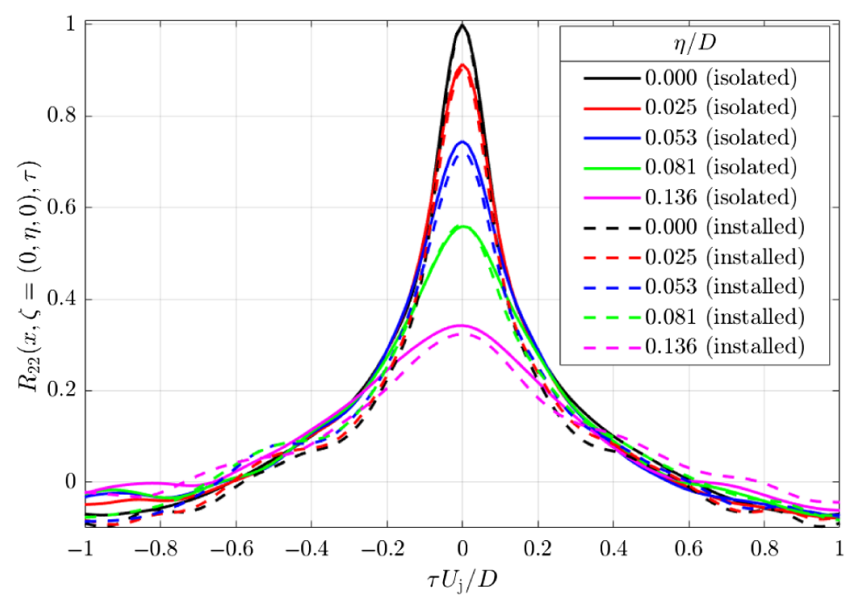

b) Radial separations $\zeta=(\mathbf{0}, \eta, 0$,

Fig. 11 Radial-velocity space-time correlation $R_{22}$ at $x / D=4$ and $r / D=0.5$ for isolated and installed jets. 
installed jets, indicating negligible nonlinear hydrodynamic interactions. However, noticeable modifications to the linear hydrodynamic and acoustic waves can be seen from near-field pressure spectra, which are discussed in the next section.

\section{Near-Field Spectra}

Comparisons are made between the experimental measurements and LES predictions to validate both the near-field velocity and pressure for the isolated jet. The velocity is normalized by the nozzle exit jet velocity for LES and experiment. Two velocity spectra are plotted in Fig. 12a at the two locations $x / D=2$ and 8 along the nozzle lip line $r \bar{D}=0.5$ : one before and the other after the potential core end. The two velocity spectra cross at around $S t=0.25$. This is because large-scale turbulence structures are generated after the jet potential core collapses, leading to an increase of low-frequency energy. The decay exponents of these turbulence spectra are around $-5 / 3$ at high frequencies. This indicates that this flow has an inertial range that is close to the scaling of isotropic turbulence. The LES captures this spectral shape and the same crossing point as those of the hot-wire measurements.

The near-field pressure spectra at $x / D=2$ for two radial positions, $r / D=1$ and 2, are compared for the isolated jet in Fig. 12b. Excellent agreement is achieved between microphone measurements and LES predictions. There are two regimes in these pressure spectra. The hump at low frequencies $(S t<1)$ is caused by hydrodynamic

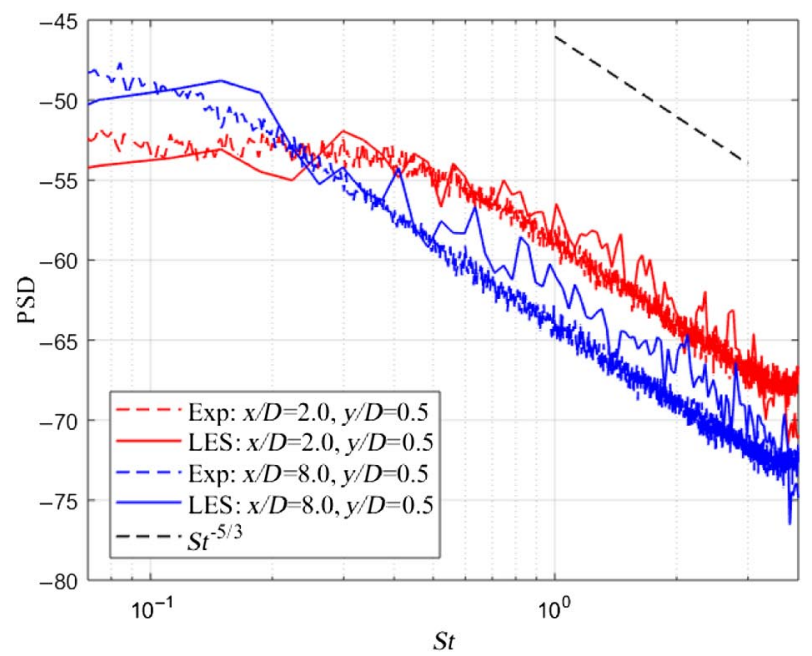

a) Velocity spectra at $x / D=2,8$ and $r / D=0.5$ evanescent waves, whereas the high frequencies $(S t>1)$ correspond to acoustics. As they move out radially, the hydrodynamic evanescent waves decay exponentially and the acoustic waves decay with $1 / r^{2}$.

For the installed jet, pressure spectra on the lower side of the plate surface are shown in Fig. 13. The LES predictions are compared with experimental measurements at three probe locations. Overall, encouraging agreement is found between the LES and experimental measurements. The spectra shape and the trends of spectra shape change with probe location are well captured by the LES. Figure $13 \mathrm{~b}$ shows a wavenumber decomposition of pressure fluctuations for each frequency along the plate surface center line. Clearly, two propagation regimes can be seen from the plot, separated by a sonic line. As indicated by the acoustic dispersion relationship between wavenumber and frequency [24], the energy above the sonic line corresponds to the waves of a phase speed larger than the sound speed $c_{0}$. These waves are acoustic, which are able to propagate to the far field. The energy under the sonic line corresponds to waves with a subsonic phase velocities, which are related to the evanescent hydrodynamic waves.

Because near-field pressure azimuthal modes can be used to model scattering noise [6], they are compared using the LES data of isolated and installed jets at the axial location of the plate TE $(x / D=4)$. The azimuthal decomposition of the near-field pressure spectra is calculated as

$$
\left.\hat{p}(x, r, m, f)=\int_{0}^{T} \int_{-\pi}^{\pi} p(x, r, \theta, t) e^{i(m \theta-2 \pi f t}\right) \mathrm{d} \theta \mathrm{d} t
$$

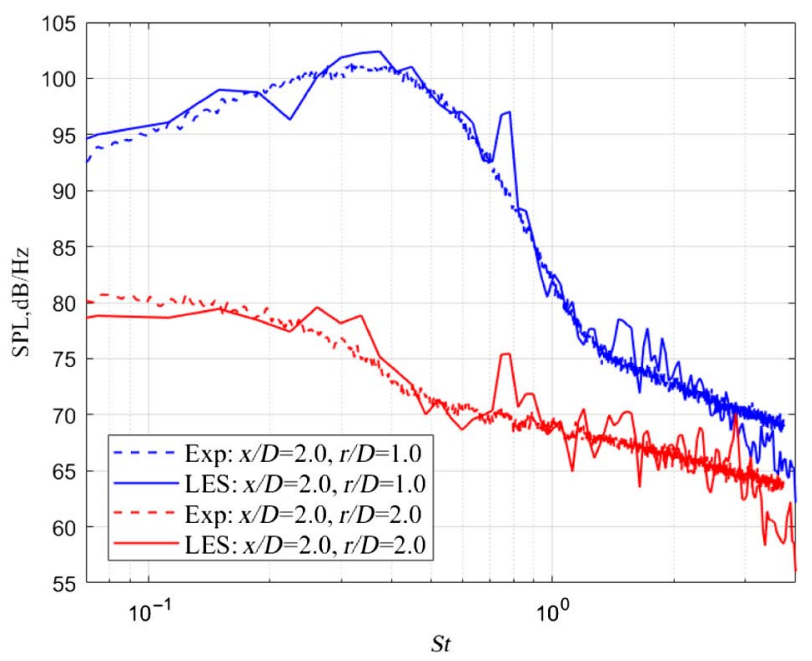

b) Pressure spectra at $x / D=2$ and $r / D=1,2$

Fig. 12 Velocity and pressure spectra in the near-field of isolated jet.

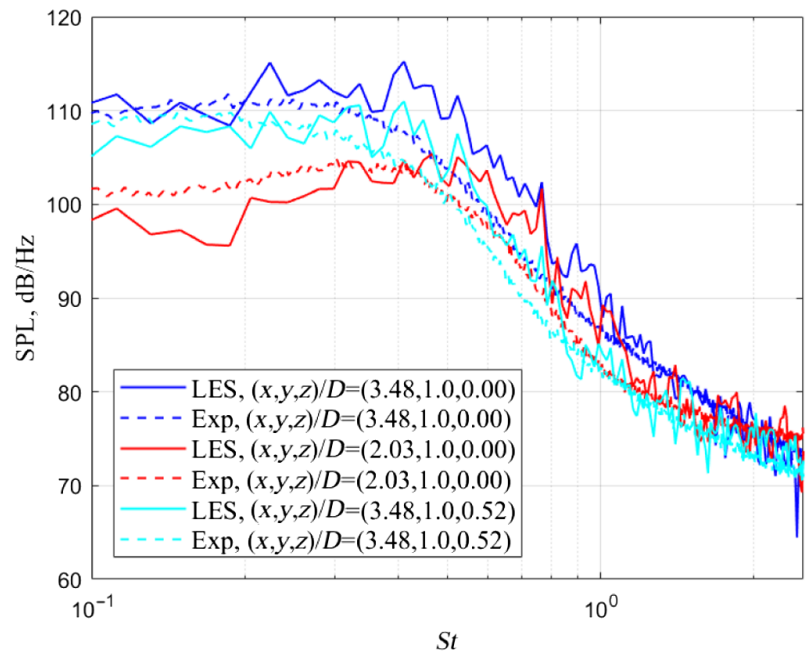

a) Frequency spectra of surface pressure

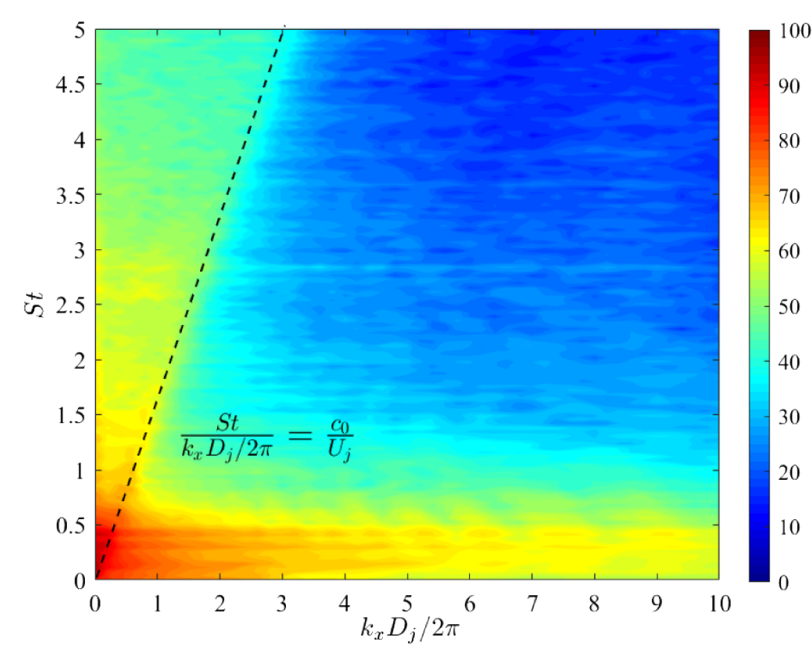

b) Wavenumber spectra of surface pressure

Fig. 13 Frequency and wavenumber spectra of pressure on the plate surface. 
where $T$ is the total sampling length, $m$ is azimuthal mode number, and $f$ is frequency. The power spectral density of the first four azimuthal modes is shown in Fig. 14 with normalization by the reference acoustic pressure level, $\overline{p_{\text {ref }}}=2 \mu \mathrm{Pa}$. The azimuthal modes are significantly amplified in the midfrequency range $0.1<S t<1$ as the hydrodynamic waves are scattered by the plate TE. The magnitude of amplification tends to increase toward the higher azimuthal modes. Figure 14a shows the spectra of the first four azimuthal modes. The amplitudes of azimuthal modes $m=2$ and 3 of installed jets reach around the same level as those of the mode $m=0$ and 1 of the isolated jets at around $S t=0.4$. Higher azimuthal modes of the near-field pressure spectra become important when the plate TE is introduced in the linear hydrodynamic region. This is further illustrated in Fig. 14b, as more energy is distributed into higher azimuthal modes between $S t=0.1$ and 1 .

\section{E. Far-Field Sound}

The far-field noise is predicted using the FW-H method for both jets. The observer angle $\phi$ is defined in Fig. 2 . Figure 15 shows the sound spectra in the far-field at two polar observer angles $\phi=40$ and $90^{\circ}$. The acoustic measurement of installed jet was performed at $M a=0.5$, and the mixing and scattering noise are scaled to $M a=0.6$ following $M a^{8}$ and $M a^{4}$ at polar angle $90^{\circ}$, respectively, and joined together at $S t=3$. Uncertainties from both $M a$ scaling and acoustic measurements are plotted in error bars. In the region between $S t=0.3$ and 0.5 , the mixing and scattering noise contribute at a similar level, and therefore simple scaling with $M a^{8}$ results in larger uncertainties. The predicted far-field sound agrees with the experimental measurements within the uncertainty range for installed jets at $\phi=90^{\circ}$. The underprediction of isolated jet noise at low frequencies at $\phi=90^{\circ}$ could be caused by inconsistent inlet turbulence levels between the LES and experiment, as well as short sampling length. This is part of our future research. Obvious installation effects are seen at $\phi=90^{\circ}$ in Fig. $15 \mathrm{~b}$. At low frequencies 10 $(S t<0.5)$, a large SPL increase at low frequencies in excess of $10 \mathrm{~dB}$ can be observed compared with the isolated jet. This noise increase is caused by the jet-surface interaction source. LES prediction captures this jet-surface interaction noise very well. At high frequencies $(S t>0.5)$, noise is shielded above the plate, which results in a lower SPL, and when noise is reflected under the plate, it leads to a slightly higher SPL. LES predictions match the experimental data at both shielded and unshielded sides in high-frequency range $(S t>0.5)$ with a mesh resolution limit up to around $S t=2$. At the lower observer angles $\phi=40^{\circ}$, only isolated jet noise measurements are available. Good agreement has also been achieved between the LES and the experimental data. The jet-alone noise, however, is dominant in this observation angle, and the installation effects become less obvious as reported by other researchers [2].

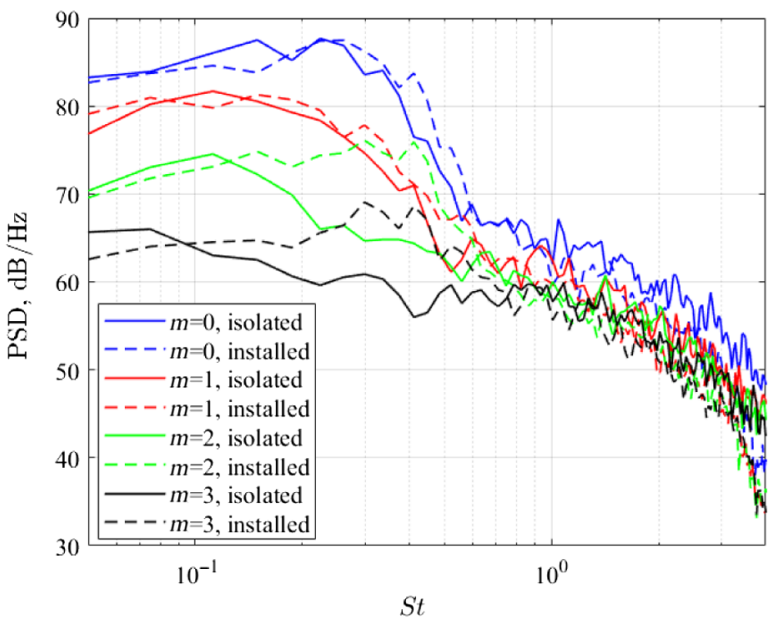

a) First four leading azimuthal modes

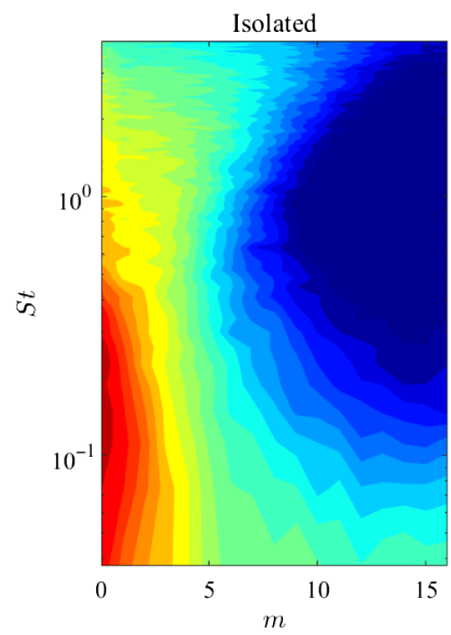

b) Azimuthal mode spectra

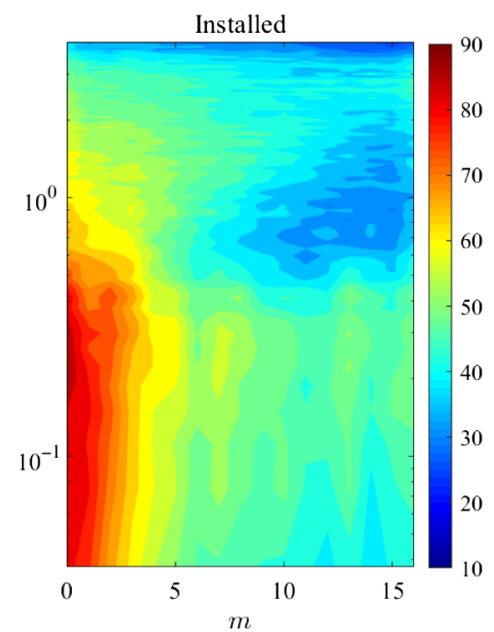

$m$

Fig. 14 Azimuthal modes of near-field pressure at $x / D=4$ and $r / D=2$ for both isolated and installed jets.

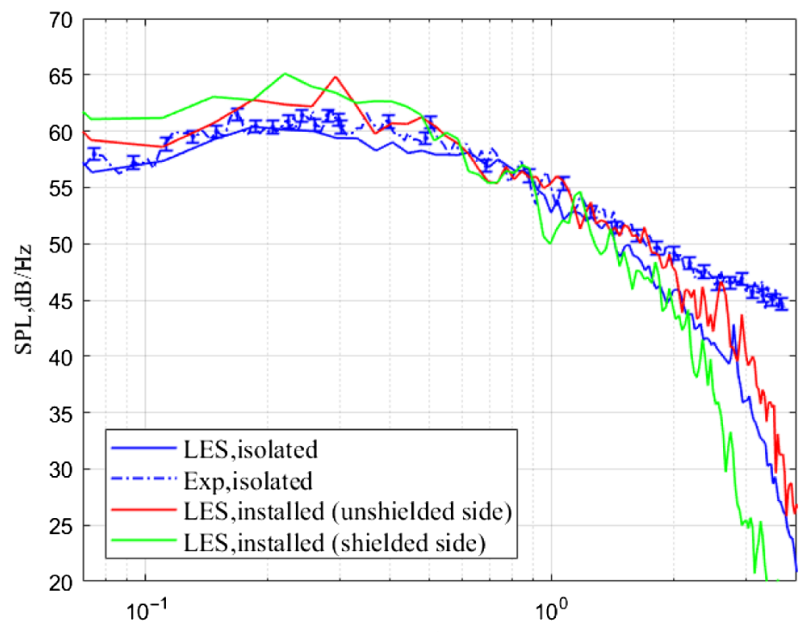

a) $\phi=40^{\circ}$

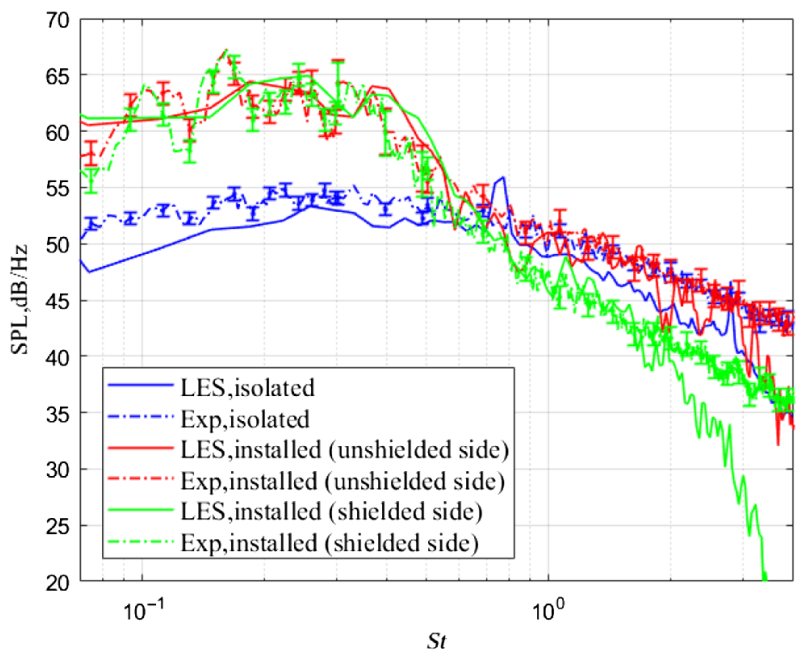

b) $\phi=90^{\circ}$

Fig. 15 Far-field sound spectra at the polar angles of $\phi=40$ and $90^{\circ}$ for the isolated and installed jets. 


\section{Conclusions}

In this paper, LES has been successfully performed on both the isolated and installed jets to investigate installation effects. Validation has been achieved by comparing LES thoroughly with experimental data from both hot-wire flow and microphone pressure measurements. Regarding the flow statistics, the LES shows a good agreement with experimental data in axial velocity profiles at $x / D=0,2,4,6,8$, and 10 . The entrainment of the jet flow is seen to be restricted by the presence of the plate, and the turbulent fluctuations are slightly suppressed in the vicinity of the plate TE. It is found in general that the installation effects are relatively small in the nearfield flow statistics for this particular jet installation configuration: $L / D=4$ and $H / D=1$. This is also confirmed by the space-time correlations of axial and radial velocities. Space-time correlations are validated by the hot-wire measurements. The LES slightly underpredicts some correlation peak values but well captures the peak locations for each spatial separation. For near-field spectra, LES captures the shape of velocity spectra and agrees well with experimentally measured pressure spectra. Using LES data, azimuthal and wavenumber decompositions were performed on the near-field pressure spectra. The azimuthal decomposition indicates that the contributions from higher azimuthal modes increase at the frequency range of $0.1<S t<1$ when the plate is present. The wavenumber decomposition along the center line of plate undersurface distinguishes the energy distributions between the propagating acoustic and evanescent hydrodynamic waves near the TE. For the far-field sound, the installation effects are obvious at the observer polar angles of $\phi=90^{\circ}$. The LES accurately predicts the jet-surface interaction noise at the low frequencies $S t<0.5$ as well as the plate shielding and reflection effects at the high frequencies $0.5<S t<2$. This well-validated LES database will be used in the future to understand installed jet noise mechanisms and inform low-order model development.

\section{Appendix: Experimental Uncertainty}

Regarding aerodynamic CTA measurements, calibration equipment, instrumentation, and experimental conditions play a role in the uncertainty of a velocity sample. The hot-wire calibration is one of the major sources of systematic error. A dedicated, automatic calibrator was used in this work. The error associated with the calibrator unit suggested by the manufacturer is less than $\pm 0.5 \%$ of the nominal velocity. Additionally, there is also uncertainty related to the linearization of the calibration data. A quartic polynomial was used to fit the data, and an error analysis was performed. It has been seen that the error is lower than $\pm 0.5 \%$ of the nominal velocity for all points measured during the calibration procedure.

The uncertainties regarding instruments used to measure ambient and flow properties (e.g., jet and chamber static pressures, temperatures, and relative humidities) are of less importance in comparison to the uncertainties discussed above. In this category, errors due to temperature variations during an experiment are dominant. Flow temperature fluctuations were measured using a thermometer mounted close to the hot-wire probe. It is expected that errors due to variations in flow and ambient properties do not exceed $1 \%$ of the sample velocity [25]. Therefore, the total error intrinsic to the CTA system is taken to be within $\pm 1.5 \%$ of the sample velocity. The uncertainty of the second-order moment (e.g., turbulence intensity) due to systematic errors is then $\pm 3 \%$.

Probe positioning is another source of uncertainty. The traverse system and the hot-wire probe holder were aligned to the jet nozzle using a laser pointer. The laser pointer was mounted parallel to the jet nozzle, and the traverse position was adjusted continuously until the hot-wire probe was seen to traverse 10 jet diameters along the laser beam axis. Several mean velocity profiles ranging from the nozzle exit to 10 diameters downstream were used to estimate the difference between the jet geometric axis and the measured axis. It was then seen that the difference between measured and geometric axis was 0.5 ॰ This angle was used to correct the position of the hot wire, and so the uncertainty associated with probe positioning is small. More information about the traverse alignment procedure and definition of geometric and measured axis are presented by Proenca ([22] Secs. 3.1.2 and 3.2.3).

The errors discussed above are in agreement with the relative expanded uncertainty in a velocity sample equals to $\pm 1.5 \%$ suggested by the CTA manufacturer. To verify this assumption, data from three different campaigns were compared. The three sets of data were acquired in a span of 4 years, but the same nozzle and traverse systems were used in all three tests. In Fig. A1, mean velocity and turbulence intensity profiles from these three campaigns are shown. Data shown as black circles were acquired using a single-wire calibrated with a manual calibrator, red crosses were obtained using a cross-wire and automatic calibrator, and blue triangles used a single-wire calibrated with the same automatic calibrator. All profiles shown were measured at $x / D=8$, as positioning errors are more obvious here than in positions close to the jet nozzle exit. It is seen from these plots and analysis of profiles at other axial locations that the variation of the mean velocity and the root mean square of the velocity fluctuations are within $\pm 1.5 \%$ in respect to the jet exit velocity. The uncertainties are plotted at $y / D=0$ in Figs. 7a and $\underline{8}$ a. The measurement errors are almost negligible for mean velocity.

Regarding the unsteady wall pressure data and the far-field acoustic measurement, repeat tests were conducted revealing a repeatability error of $\pm 0.5 \% \mathrm{~dB}$ across the entire frequency range of interest. As for the microphone hardware itself, the systematic errors add up to less than $\pm 0.05 \% \mathrm{~dB}$. The $M a$ scaling of scattering and mixing noise leads to an uncertainty of $\pm 0.5 \% \mathrm{~dB}$. In the joint region of these two types of noise spectra, $0.3 \leq S t \leq 0.5$, the scaling uncertainties are within $\pm 2 \% \mathrm{~dB}$. This is estimated by comparing the scaling of $M a^{4}$ for scattering noise and $M a^{8}$ for mixing noise.

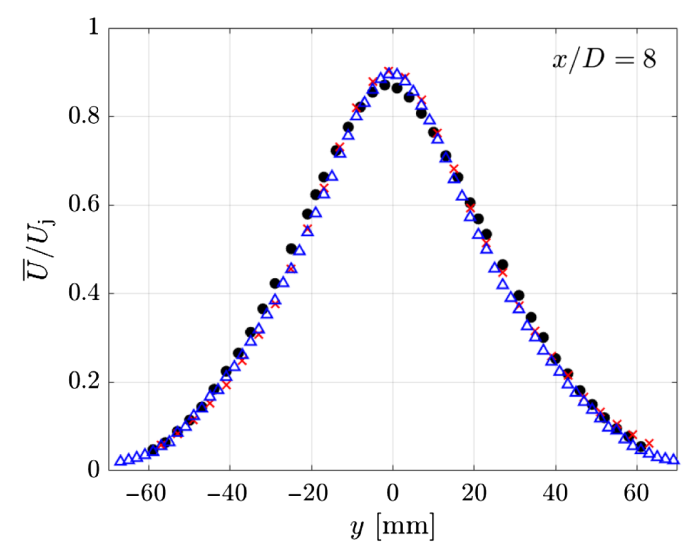

a) Mean velocity

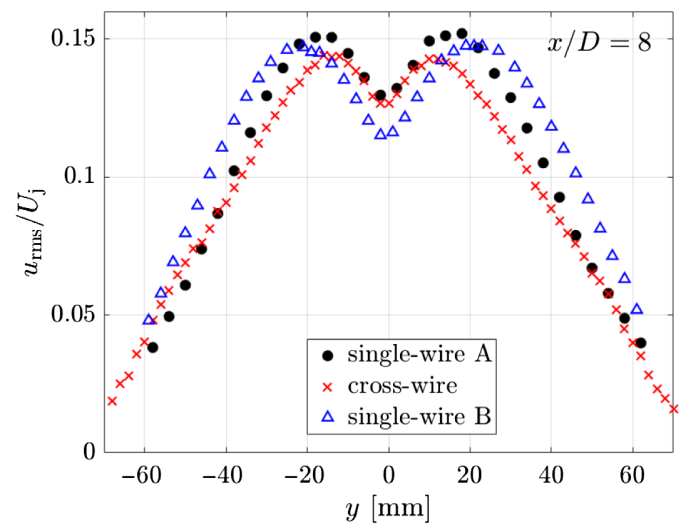

b) Turbulence intensity

Fig. A1 a) Mean velocity and b) turbulence intensity of an $M=0.6$ jet measured in three different experimental campaigns at the Doak Laboratory. 


\section{Acknowledgments}

Acknowledgement is made for the computational time provided by the U.K. Turbulence Consortium under EPSRC grant EP/L000261/1 on U.K. national supercomputer ARCHER and the DECI resource Sisu based in Finland with support from the PRACE aisbl under the project InJet.

\section{References}

[1] Lighthill, M. J., "On Sound Generated Aerodynamically II. Turbulence as a Source of Sound," Proceedings of the Royal Society of London. Series A. Mathematical and Physical Sciences, Vol. 222, No. 1148, 1954, pp. 1-32.

[2] Head, R., and Fisher, M., "Jet Surface Interaction Noise - Analysis of Far-Field Low Frequency Augmentations of Jet Noise Due to the Presence of a Solid Shield," AIAA Paper 1976-502, 1976.

[3] Bushell, K., "Measurement and Prediction of Jet Noise in Fight," AIAA Paper 1975-461, 1975.

[4] Fisher, M., Harper-Bourne, M., and Glegg, S., "Jet Engine Noise Source Location: The Polar Correlation Technique," Journal of Sound and Vibration, Vol. 51, No. 1, 1977, pp. 23-54. https://doi.org/10.1016/S0022-460X(77)80111-9

[5] Williams, J. E. F., and Hall, L. H., "Aerodynamic Sound Generation by Turbulent Flow in the Vicinity of a Scattering Half Plane," Journal of Fluid Mechanics, Vol. 40, No. 4, 1970, pp. 657-670. https://doi.org/10.1017/S0022112070000368

[6] Lyu, B., Dowling, A. P., and Naqavi, I., "Prediction of Installed Jet Noise," Journal of Fluid Mechanics, Vol. 811, Jan. 2017, pp. 234-268. https://doi.org/10.1017//fm.2016.747

[7] Amiet, R. K., "Acoustic Radiation from an Airfoil in a Turbulent Stream," Journal of Sound and Vibration, Vol. 41, No. 4, 1975, pp. 407-420. https://doi.org/10.1016/S0022-460X(75)80105-2

[8] Bodony, D. J., and Lele, S. K., "Current Status of Jet Noise Predictions Using Large-Eddy Simulation,” AIAA Journal, Vol. 46, No. 2, 2008, pp. 364-380. https://doi.org/10.2514/1.24475

[9] Wang, Z.-N., Tyacke, J. C., Tucker, P. G., and Boehning, P., "Parallel Computation of Aeroacoustics of Industrially Relevant ComplexGeometry Aeroengine Jets," Computers \& Fluids, Vol. 178, No. 2, 2019, pp. 166-178. https://doi.org/10.1016/j.compfluid.2018.04.039

[10] Suzuki, T., and Colonius, T., "Instability Wave in a Subsonic Round Jet Detected Using a Near-Field Phased Microphone Array," Journal of Fluid Mechanics, Vol. 565, Oct. 2006, pp. 197-226. https://doi.org/10.1017/S0022112006001613

[11] Tyacke, J. C., Wang, Z.-N., and Tucker, P. G., "LES-RANS of Installed Ultra-High-Bypass-Ratio Coaxial Jet Aeroacoustics with Flight Stream," AIAA Journal, Vol. 57, No. 3, 2019, pp. 1215-1236. https://doi.org/10.2514/1.J057057

[12] Bridge, J., and Wernet, M. P., "Validating Large-Eddy Simulation for Jet Aeroacoustics," Journal of Propulsion and Power, Vol. 28, No. 2, 2012, pp. 226-235.

https://doi.org/10.2514/1.B34385
[13] Jameson, A., "Formulation of Kinetic Energy Preserving Conservative Schemes for Gas Dynamics and Direct Numerical Simulation of OneDimensional Viscous Compressible Flow in a Shock Tube Using Entropy and Kinetic Energy Preserving Schemes," Journal of Scientific Computing, Vol. 34, No. 2, 2008, pp. 188-208. https://doi.org/10.1007/s10915-007-9172-6

[14] Nicoud, F., and Ducros, F., "Subgrid-Scale Stress Modelling Based on the Square of the Velocity Gradient Tensor," Flow, Turbulence, and Combustion, Vol. 62, No. 3, 1999, pp. 183-200. https://doi.org/10.1023/A:1009995426001

[15] Spalart, P. R., and Allmaras, S. R., "A One-Equation Turbulence Model for Aerodynamic Flows," La Recherche Aerospatiale, Vol. 1, 1994, pp. 5-21.

[16] Wang, Z. N., Tyacke, J. C., Tucker, P. G., and Boening, P., "Guide for Conducting Jet Noise Simulation with LES/DES in Hydra (How-toGuide)," Rolls-Royce Deutschland Ltd \& Co KG, ED-63/M/14-DW, 2017, (circa 14 pages).

[17] Mahak, M., Naqavi, I., and Tucker, P. G., "Cost-Effective Hybrid RANSLES Type Method for Jet Turbulence and Noise Prediction," International Journal of Aeroacoustics, Vol. 16, Nos. 1-2, 2017, pp. 97-111. https://doi.org/10.1177/1475472X16684702

[18] Bres, G. A., Jordan, P., Jaunet, V., Le Rallic, M., Cavalieri, A. V. G., Towne, A., Lele, S. K., Colonius, T., and Schmidt, O. T., "Importance of the Nozzle-Exit Boundary-Layer State in Subsonic Turbulent Jets," Journal of Fluid Mechanics, Vol. 851, Sept. 2018, pp. 83-124. https://doi.org/10.1017/jfm.2018.476

[19] Najafi-Yazdi, A., Bres, G. A., and Mongeau, L., "An Acoustic Analogy Formulation for Moving Sources in Uniformly Moving Media," Proceedings of the Royal Society of London. Series A. Mathematical and Physical Sciences, Vol. 467, No. 2125, 2010, pp. 1-22.

[20] Bass, H. E., Sutherland, L. C., Zuckerwar, A. J., Blackstock, D. T., and Hester, D. M., "Atmospheric Absorption of Sound: Further Developments," Journal of the Acoustical Society of America, Vol. 97, No. 1, 1995, pp. 680-683. https://doi.org/10.1121/1.412989

[21] Lawrence, J., "Aeroacoustic Interactions of Installed Subsonic Round Jets," Ph.D. Thesis, Univ. of Southampton, Southampton, England, U.K., 2014.

[22] Proenca, A., "Aeroacoustics of Isolated and Installed Jets Under Static and In-Flight Conditions," Ph.D. Thesis, Univ. of Southampton, Southampton, England, U.K., 2018.

[23] Proenca, A., Lawrence, J., and Self, R., "Measurements of the Single-Point and Joint Turbulence Statistics of High Subsonic Jets Using Hot-Wire Anemometry," Experiments in Fluids, Vol. 64, No. 4, 2019, pp. 1432-1114.

[24] Dowling, A. P., and Hynes, T. P., "Sound Generation by Turbulence," European Journal of Mechanics B/Fluids, Vol. 23, No. 3, 2004, pp. 491-500. https://doi.org/10.1016/j.euromechflu.2003.10.014

[25] Jørgensen, F. E., "How to Measure Turbulence with Hot-Wire Anemometers - A Practical Guide," Tech. Rept., Dantec Dynamics, Skovlunde, Denmark, 2005.

C. Bailly

Associate Editor 


\section{Queries}

1. AU: Please check that the copyright (@) type is correct. Please note that the code will be added upon publication.

2. AU: LES has been expanded in the title per AIAA style. Please check.

3. AU: Nomenclature entries have been reordered and edited per the style of AIAA. Please check.

4. AU: Acronym UHBP has been changed to UHBR to match the expansion. Please check.

5. AU: Please define WALE SGS.

6. AU: Please expand MPI.

7. AU: Please expand I/O.

8. AU: Please expand HDF5.

9. AU: As AIAA displays vectors in bold-italic font, some of the variables have been edited. Please confirm that all variables are displayed correctly throughout the paper. Please ensure that, throughout the paper, vector symbols are in bold-italic font, and that nonvector variables are in italic font. If any of the vector symbols are NOT currently in bold-italic font in the proofs, please mark them clearly so that we can make them bold-italic.

10. AU: Please define SPL.

11. AU: The sentence "At high frequencies ( $t>0.5)$, noise is shielded ..." has been rephrased for clarity. Please check if your meaning is retained.

12. AU: The sentence "The errors discussed above are in agreement with the relative ..." is not clear. Please rephrase.

13. AU: The sentence "as positioning errors are more obvious here than in positions close to the jet nozzle exit ..." has been rephrased for clarity. Please check if your meaning is retained.

14. AU: Please expand EPSRC, ARCHER, DECI, Sisu, and PRACE aisbl in the Acknowledgments.

15. AU: Please provide issue number or month of publication for Ref. [15].

16. AU: Please provide institution location for Ref. [16].

17. AU: Please provide report number for Ref. [25].

\section{Funding Information}

The following research funding sources have been associated with your manuscript:

- EPSRC; Award no. EP/L000261/1

- Partnership for Advanced Computing in Europe AISBL; Award no. DECI InJet;

Funder ID http://dx.doi.org/10.13039/501100001943

Funding sources listed here can be 1) research grants from outside agencies or organizations or 2) if an author is employed by a U.S. government agency that directly funded this research, this employer also may be listed here as a funding agency. Please confirm that 
this information is complete and correct for all authors. Edit the Funding Data/Acknowledgments section of your paper if you have changes to funding agency names or grant numbers. Use the funder's full and official name. 